\title{
A Comparative Study of q-Homotopy Analysis Method and Liao's Optimal Homotopy Analysis Method
}

\author{
Shaheed N. Huseen \\ Mathematics Department, Faculty of Computer Science and Mathematics, University of Thi-Qar, Thi-Qar, Iraq.
}

\author{
How to cite this paper: Shaheed N. Hu- \\ seen. (2020) A Comparative Study of \\ q-Homotopy Analysis Method and Liao's \\ Optimal Homotopy Analysis Method. Ad- \\ vances in Computer and Communication, \\ 1(1), 36-45. \\ DOI: $10.26855 /$ acc.2020.12.004 \\ Received: October 30, 2020 \\ Accepted: November 28, 2020 \\ Published: December 15, 2020 \\ *Corresponding author: Shaheed N. \\ Huseen, Mathematics Department, Fa- \\ culty of Computer Science and Mathe- \\ matics, University of Thi-Qar, Thi-Qar, \\ Iraq. \\ Email: shn_n2002@yahoo.com
}

\begin{abstract}
In this paper, comparative study of q-homotopy analysis method (q-HAM) with the Liao's optimal homotopy analysis method (OHAM) is proposed. We solved two examples, first example is a system of Volterra integro-differential equations and the second one is a nonlinear integro-differential equation. The results show that the q-HAM was more accuracy than the OHAM.
\end{abstract}

\section{Keywords}

q-homotopy analysis method, Convergence parameter, Integro-differential equations

\section{Introduction}

A numerous methods were proposed to find approximate solutions for nonlinear phenomena of our life. Liao [1-5] proposed an analytic method for solving linear and nonlinear problems, namely homotopy analysis method (HAM). Recently, HAM has been successfully applied to solve different types of non-linear problems in variety fields [6, 7, 8, 9, 10]. HAM contains a convergence parameter $h$ which with a simple way provides us to adjust and control the convergence region of the series solution. Moreover, by means of h-curve, easily to gain the valid regions of $h$ to find a convergent series solution. We cannot determine the best value of $h$ by plotting the h-curves, to find the fastest convergent series. Yabushitaet al. [11] suggested the "optimization method" to find out the two optimal values of $h$ by the minimum of the squared residual error. Akyildiz and Vajravelu [12] determine an optimal value of $h$ by the minimum of squared residual and found that the homotopy-series solution converges so quickly. A one-step optimal homotopy analysis method was proposed by Niu and Wang [13]. Liao [14] introduced an optimal HAM with three convergence parameters. A general method of HAM namely q-homotopy analysis method (q-HAM) was proposed by El-Tawil and Huseen [15], the q-HAM contains two convergence parameters $n$ and $h$ such that the case of $n=1$ the HAM can be reached. Many researches applied the q-HAM to numerous problems in different felids [16-28].

\section{Fundamental Idea ofq-HAM}

Consider the following differential equation: 


$$
N[u(t)]=0,
$$

where $N$ is a nonlinear operator, $u(t)$ is an unknown function. The zero-order deformation equation of q-HAM is

$$
(1-n q) L\left[\varnothing(t, q)-u_{0}(t)\right]=q h N[\varnothing(t, q)]
$$

where $\geq 1,0 \leq q \leq \frac{1}{n}$ is the embedded parameter, $L$ is a linear operator and $h \neq 0$.

If $q=0$ and $q=\frac{1}{n}$ then equation (2) becomes

$$
\varnothing(t, 0)=u_{0}(t), \quad \varnothing\left(t, \frac{1}{n}\right)=u(t)
$$

Respectively. Thus as $q$ varies from 0 to $\frac{1}{n}$, the solution $\emptyset(t, q)$ varies from the initial guess $u_{0}(t)$ to the solution $u(t)$.

Taking the Taylor series of $\emptyset(t, q)$ we obtain

$$
\varnothing(t, q)=u_{0}(t)+\sum_{m=1}^{+\infty} u_{m}(t) q^{m},
$$

where

$$
u_{m}(t)=\left.\frac{1}{m !} \frac{\partial^{m} \varnothing(t, q)}{\partial q^{m}}\right|_{q=0}
$$

Let we choose $h, u_{0}(t), L$ such that the series (4) converges at $q=\frac{1}{n}$ and

$$
u(t)=\varnothing\left(t, \frac{1}{n}\right)=u_{0}(t)+\sum_{m=1}^{+\infty} u_{m}(t)\left(\frac{1}{n}\right)^{m}
$$

Defining the vector $u_{r}(t)=\left\{u_{0}(t), u_{1}(t), u_{2}(t), \ldots, u_{r}(t)\right\}$, differentiating Eq. (2) $m$ times with respect to $q$ and then setting $q=0$ and dividing by $m$ ! we have the $m^{\text {th }}$ order deformation equation

$$
L\left[u_{m}(t)-k_{m} u_{m-1}(t)\right]=h R_{m}\left(\overrightarrow{u_{m-1}}(t)\right),
$$

where

$$
R_{m}\left(\overrightarrow{u_{m-1}}(t)\right)=\left.\frac{1}{(m-1) !} \frac{\partial^{m-1} N[\varnothing(t, q)]}{\partial q^{m-1}}\right|_{q=0},
$$

and

$$
k_{m}=\left\{\begin{array}{lr}
0 & m \leq 1 \\
n & \text { otherwise }
\end{array}\right.
$$

Let

$$
\Delta_{m}=\int\left(N\left[U_{m}(t)\right]\right)^{2} d \Omega, \quad t \in \Omega,
$$

where

$$
U_{m}(t)=u_{0}(t)+\sum_{k=1}^{m} u_{k}(t)\left(\frac{1}{n}\right)^{k}
$$

is the square residual error of the $m t h$-order approximation of the Eq. (1) integrated in the whole domain $\Omega$. In theory if the square residual error $\Delta_{m}$ tends to zero, then

$$
U_{m}(t)=\sum_{k=0}^{m} u_{k}(t)\left(\frac{1}{n}\right)^{k}
$$

is a series solution of the original equation (1). Besides, at the given order of approximation, the minimum of the squared residual error $\Delta_{m}$ corresponds to the optimal approximation. So, the curves of the squared residual error $\Delta_{m}$ versus $h$ indicate to the valid region of $h$, hence the optimal value of $h$ that corresponds to the minimum of $\Delta_{m}$.

Liao [14] introduced the so-called average residual error $E_{m}=\frac{1}{K} \sum_{j=0}^{K}\left[N\left(\sum_{k=0}^{m} u_{k}(j \Delta x)\right)\right]^{2}$ where $\Delta x=\frac{1}{K}$.

\section{Applications}

Example 3.1.Consider the following system of Volterra integro-differential equations [29]

$$
u_{1}^{\prime}=1+t+t^{2}-u_{2}(t)-\int_{0}^{t}\left(u_{1}(s)+u_{2}(s)\right) d s, \quad u_{1}(0)=1,
$$




$$
u_{2}^{\prime}=-1-t+u_{1}(t)-\int_{0}^{t}\left(u_{1}(s)-u_{2}(s)\right) d s, \quad u_{2}(0)=-1,
$$

With exact solutions $u_{1}(t)=t+\exp (t)$ and $u_{2}(t)=t-\exp (t)$.

Let $L\left[\emptyset_{i}(t, q)\right]=\frac{\partial \emptyset_{i}(t, q)}{\partial t}$. From (11) the nonlinear operators are

$$
\begin{gathered}
N_{1}\left[\emptyset_{1}(t, q)\right]=\frac{\partial \emptyset_{1}(t, q)}{\partial t}-\left(1+t+t^{2}\right)+\emptyset_{2}(t, q)+\int_{0}^{t}\left(\emptyset_{1}(s, q)+\emptyset_{2}(s, q)\right) d s \\
N_{2}\left[\emptyset_{2}(t, q)\right]=\frac{\partial \emptyset_{2}(t, q)}{\partial t}+(1+t)-\emptyset_{1}(t, q)+\int_{0}^{t}\left(\emptyset_{1}(s, q)-\emptyset_{2}(s, q)\right) d s
\end{gathered}
$$

Let $u_{1,0}(t)=\exp (t)$ and $u_{2,0}(t)=-\exp (t)$

According to Eq. (2) and Eq. (7) with

$$
\begin{gathered}
R_{1, m-1}(t)=u_{1, m-1}^{\prime}-\left(1-\frac{k_{m}}{n}\right)\left(1+t+t^{2}\right)+u_{2, m-1}+\int_{0}^{t}\left(u_{1, m-1}+u_{2, m-1}\right) d s \\
R_{2, m-1}(t)=u_{2, m-1}^{\prime}-\left(1-\frac{k_{m}}{n}\right)(1+t)-u_{1, m-1}+\int_{0}^{t}\left(u_{1, m-1}-u_{2, m-1}\right) d s
\end{gathered}
$$

The solution of Eq. (7) for $m \geq 1$ becomes

Hence, we obtain

$$
u_{i, m}(t)=k_{m} u_{i, m-1}(t)+h \int R_{i, m-1}(t) d t+b_{i}, i=1,2
$$

$$
\begin{aligned}
& u_{1,1}=h\left(-t-\frac{t^{2}}{2}-\frac{t^{3}}{3}\right) \\
& u_{2,1}=h\left(-t+\frac{t^{2}}{2}\right) \\
& u_{1,2}=h n\left(-t-\frac{t^{2}}{2}-\frac{t^{3}}{3}\right)+h\left(-h t-h t^{2}-\frac{h t^{3}}{2}-\frac{h t^{5}}{60}\right) \\
& u_{2,2}=h n\left(-t+\frac{t^{2}}{2}\right)+h\left(-h t+h t^{2}+\frac{h t^{3}}{6}-\frac{h t^{5}}{60}\right)
\end{aligned}
$$

Then the series solution of q- HAM is

$$
u_{i}(t, n, h) \cong U_{i, M}(t, n, h)=\sum_{k=0}^{M} u_{i, k}(t, n, h)\left(\frac{1}{n}\right)^{k}
$$

The $4^{\text {th }}$ order Liao's optimal HAM (OHAM) approximation solutions are [29]

$$
\begin{aligned}
& U_{1,4(\text { OHAM })}=e^{t}+0.999994 t+0.000227256 t^{2}+0.00234468 t^{3}-0.00705523 t^{4}-0.0098502 t^{5} \\
& +0.000470348 t^{6}+0.000782842 t^{7}-0.00000900634 t^{9}
\end{aligned}
$$

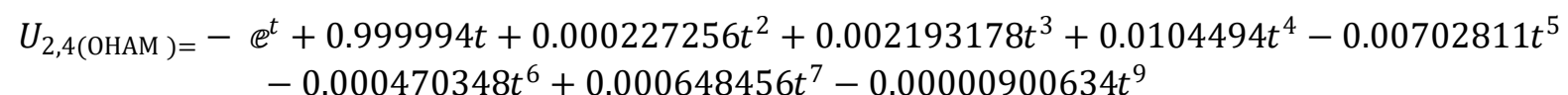

It is found that

$$
\begin{aligned}
& \triangle_{1,1}=\frac{37}{10}+\frac{336059 h^{2}}{45360 n^{2}}+\frac{3287 h}{315 n} \\
& \triangle_{2,1}=\frac{1}{3}+\frac{4687 h^{2}}{9072 n^{2}}+\frac{23 h}{90 n} \\
& \triangle_{1,2}=\frac{37}{10}+\frac{346114261 h^{4}}{32432400 n^{4}}+\frac{44355323 h^{3}}{1247400 n^{3}}+\frac{95561 h^{2}}{2268 n^{2}}+\frac{6574 h}{315 n}
\end{aligned}
$$




$$
\triangle_{2,2}=\frac{1}{3}+\frac{811787 h^{4}}{356400 n^{4}}+\frac{148529 h^{3}}{37800 n^{3}}+\frac{20969 h^{2}}{11340 n^{2}}+\frac{23 h}{45 n}
$$

$\Delta_{i, m}(t),(i=1,2),(m=3,4, \ldots)$ can be calculated similarly.

Figures $1-5$ show the $h$-curves of the square residual error $\Delta_{i, m}(i=1,2)$ given by $4^{\text {th }}$ order approximation to determine the valid region of $h$. The values of $h$ that corresponds to the minimum of the square residual errors is an optimal values of $h$.
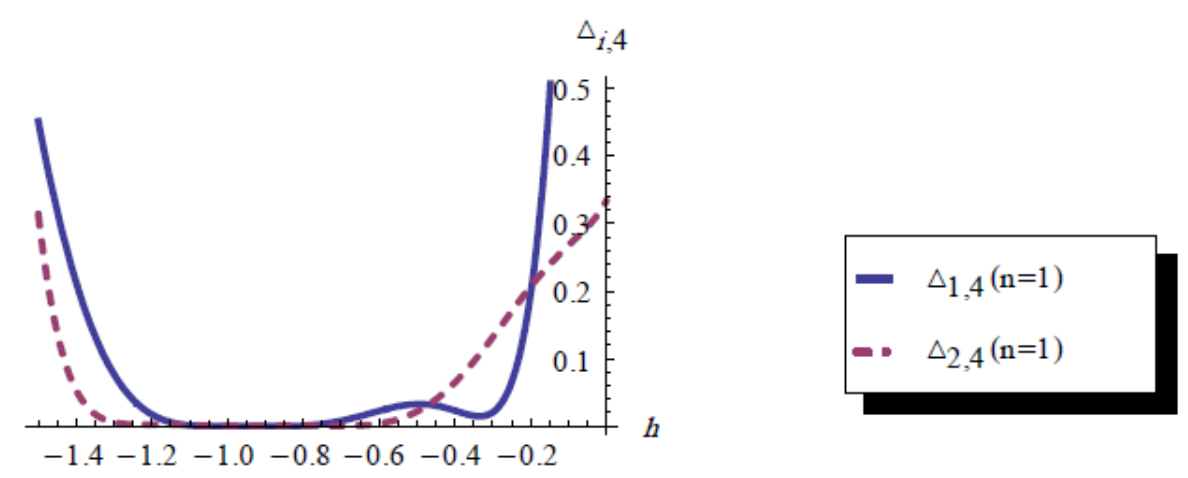

Figure 1. The residual errors $\Delta_{i, 4}(i=1,2)$ for the HAM (q-HAM; $\left.n=1\right)$ of system (11).

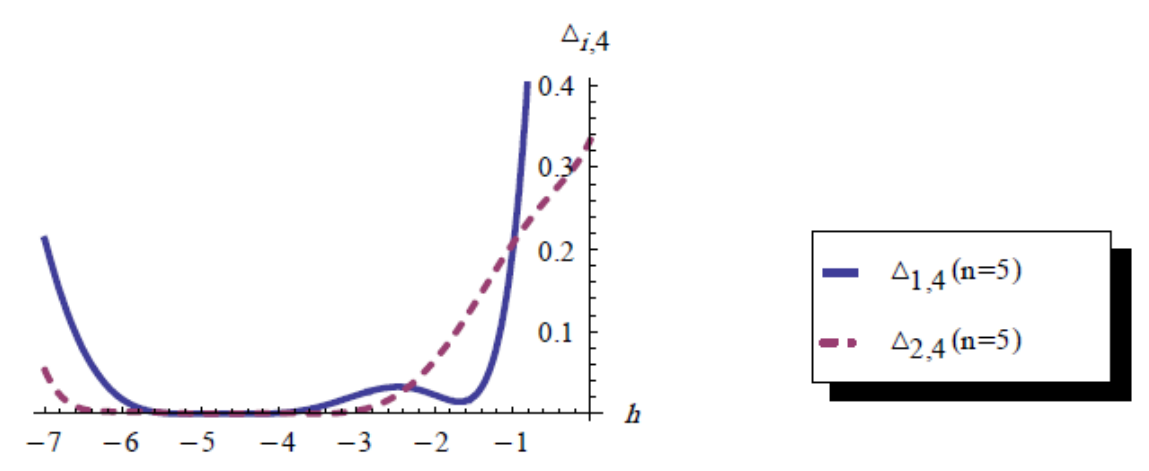

Figure 2. The residual errors $\Delta_{i, 4}(i=1,2)$ for the (q-HAM; $\left.n=5\right)$ of system (11).

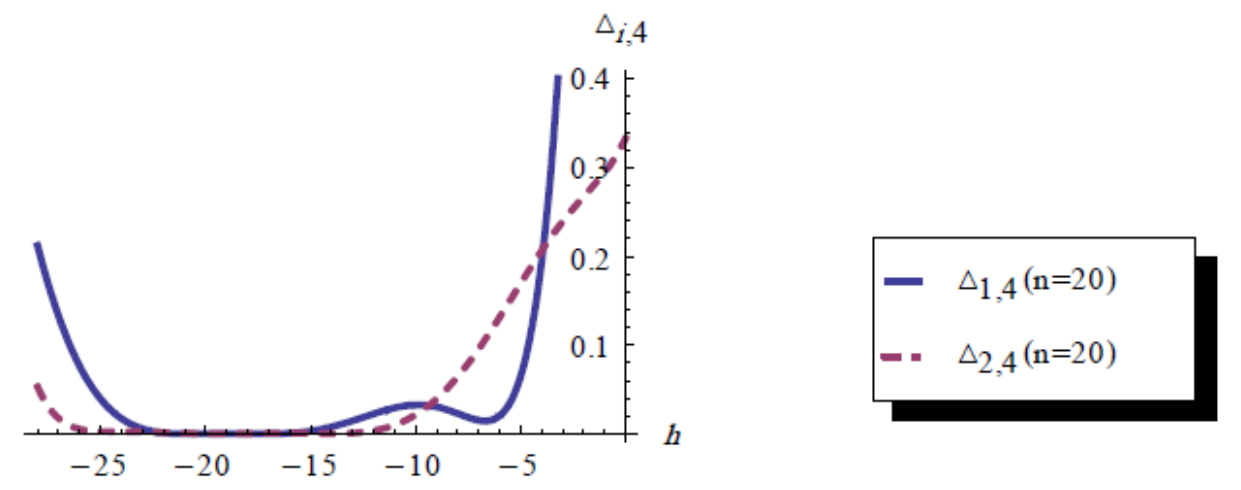

Figure 3. The residual errors $\Delta_{i, 4}(i=1,2)$ for the (q-HAM; $\left.n=20\right)$ of system (11). 


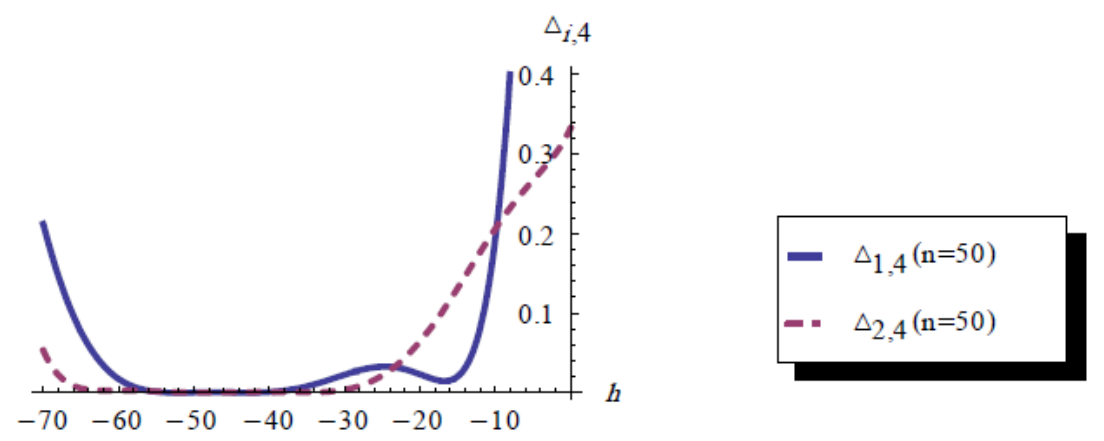

Figure 4. The residual errors $\Delta_{i, 4}(i=1,2)$ for the (q-HAM; $\left.n=50\right)$ of system (11).
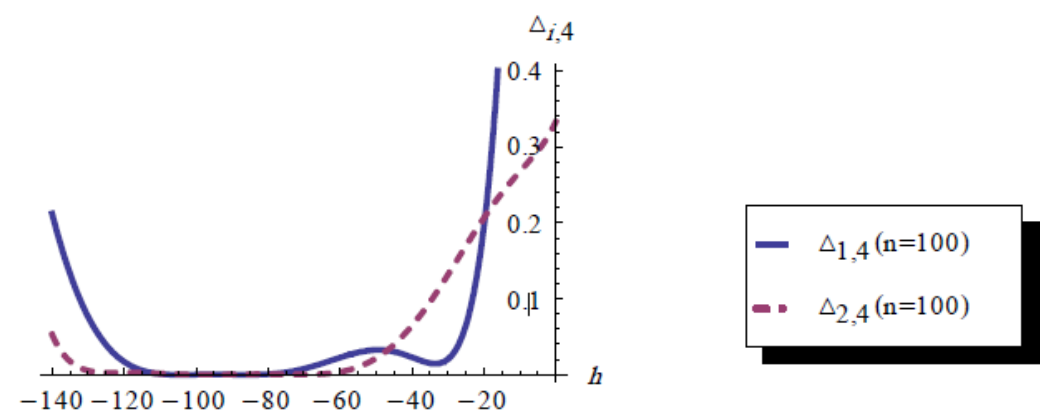

Figure 5. The residual errors $\Delta_{i, 4}(i=1,2)$ for the (q-HAM; $\left.n=100\right)$ of system (11).

Tables 1 and 2 show the comparison of $U_{1,4}, U_{2,4}$ given by Liao's optimal HAM ( OHAM), HAM (q-HAM; $n=$ 1 ) and q-HAM at different values of $n>1$ and $U_{1,6}, U_{2,6}$ given by q-HAM $(n=100)$ with the solutions $u_{1}$ and $u_{2}$. Figures (6-9) illustrate that the highly decreasing of the absolute errors by taking more terms into consideration.

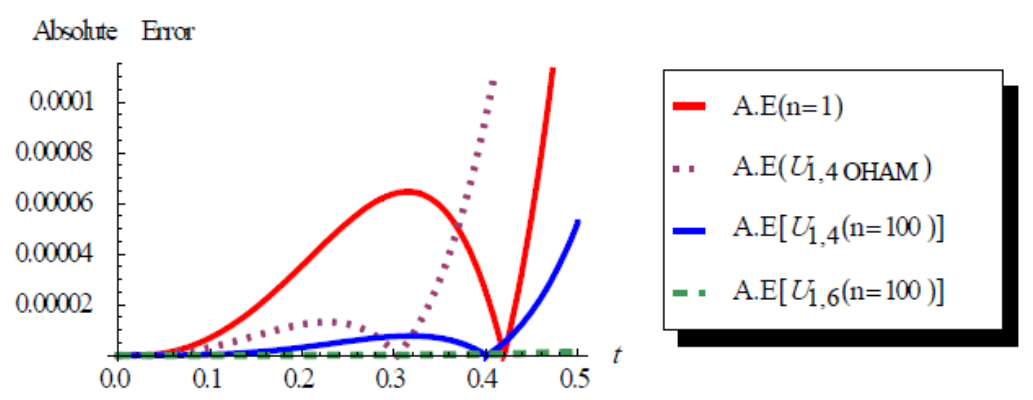

Figure 6. Absolute error of $U_{1,4}$ Liao's optimal HAM (OHAM), $U_{1,4}$ HAM (q-HAM; $\left.n=1\right), U_{1,4}$ q-HAM ( $\left.n=100\right)$ and $U_{1,6}$ q-HAM $(n=100)$ for the system (11) at $\leq t \leq 0.5$ using $(h=-0.9326, h=-101.49)$ respectively.

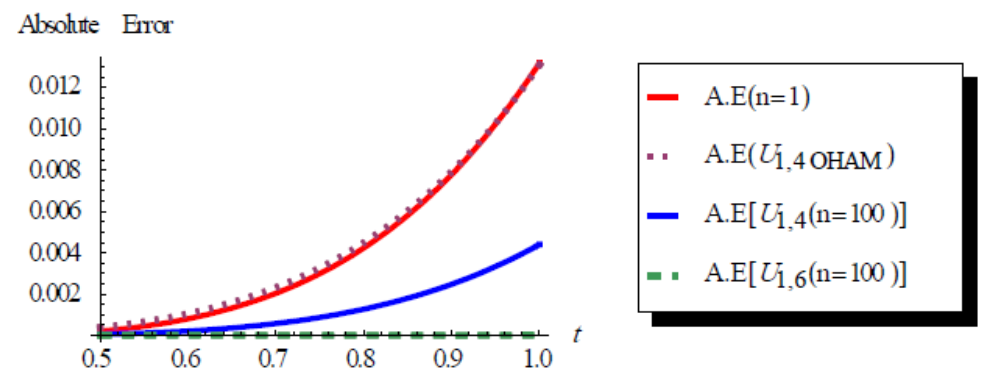

Figure 7. Absolute error of $U_{1,4}$ Liao's optimal HAM (OHAM), $U_{1,4}$ HAM (q-HAM; $\left.n=1\right), U_{1,4}$ q-HAM $(n=100)$ and $U_{1,6}$ q-HAM $(n=100)$ for the system (11) at $0.5 \leq t \leq 1$ using $(h=-0.9326, h=-101.49)$ respectively. 


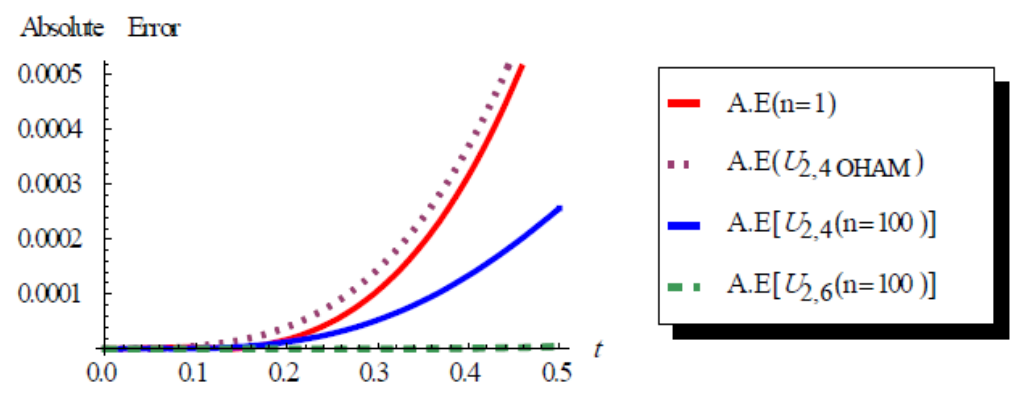

Figure 8. Absolute error of $U_{2,4}$ Liao's optimal HAM (OHAM) $U_{2,4}$ HAM (q-HAM; $\left.n=1\right), U_{2,4}$ q-HAM $(n=100)$ and $U_{2,6}$ q-HAM $(n=100)$ for the system (11) at $0 \leq t \leq 0.5$ using $(h=-0.9326, h=-96.25)$ respectively.

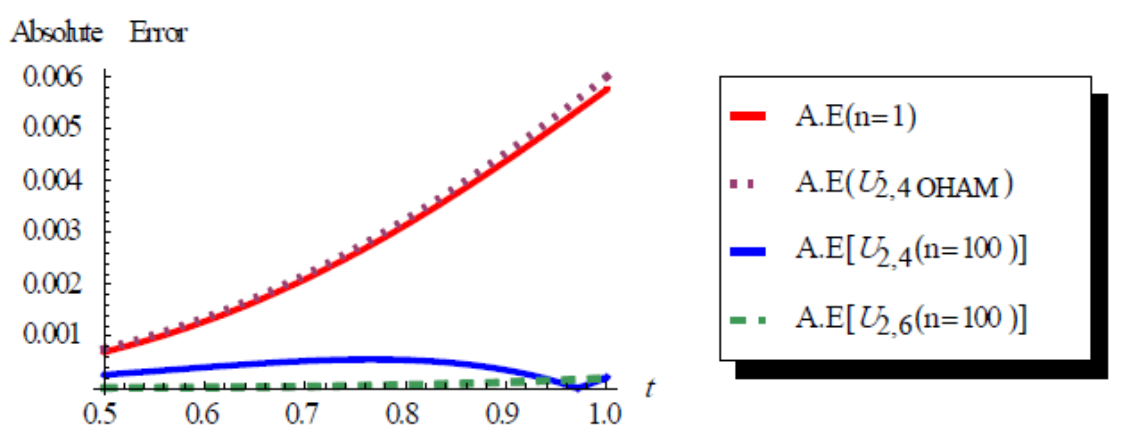

Figure 9. Absolute error of $U_{2,4}$ Liao's optimal HAM (OHAM), $U_{2,4}$ HAM (q-HAM; $\left.n=1\right), U_{2,4}$ q-HAM $(n=100)$ and $U_{2,6}$ q-HAM $(n=100)$ for the system (11) at $0 \leq t \leq 0.5$ using $(h=-0.9326, h=-96.25)$ respectively.

Table 1. Comparison of $U_{1,4}$ given by OHAM, HAM (q-HAM ; $\left.n=1\right)$, q-HAM $(n=5,20,50,100)$ and $U_{1,6}$ of q-HAM $(n=100)$ with the solution $u_{1}$

\begin{tabular}{|c|c|c|c|c|c|c|c|c|}
\hline$t$ & $\begin{array}{c}U_{1,4} \\
\text { OHAM }\end{array}$ & $\begin{array}{l}U_{1,4} \\
\quad(n=1 ; h \\
=-0.9326\end{array}$ & $\begin{array}{l}\boldsymbol{U}_{\mathbf{1}, \mathbf{4}} \\
\quad(\boldsymbol{n}=\mathbf{5} ; \boldsymbol{h} \\
=-\mathbf{5 . 0 0 1}\end{array}$ & $\begin{array}{l}U_{1,4} \\
(n=20 ; h \\
=-20.1\end{array}$ & $\begin{array}{l}U_{1,4} \\
(n=50 ; h \\
=-50.5\end{array}$ & $\begin{array}{l}U_{1,4} \\
\quad(n=100 ; h \\
\quad=-101.49\end{array}$ & $u_{1}$ & $\begin{array}{l}U_{1,6} \\
\quad(n=100 ; h \\
=-101.49\end{array}$ \\
\hline $\mathbf{0}$ & 1 & 1 & 1 & 1 & 1 & 1 & 1 & 1 \\
\hline 0.2 & 1.421412 & 1.42144 & 1.4214 & 1.4214 & 1.4214 & 1.4214 & 1.4214 & 1.4214 \\
\hline 0.4 & 1.89173 & 1.89185 & 1.89174 & 1.89177 & 1.89179 & 1.89182 & 1.89182 & 1.89182 \\
\hline 0.6 & 2.42107 & 2.42131 & 2.4215 & 2.42162 & 2.42176 & 2.42191 & 2.42212 & 2.42212 \\
\hline 0.8 & 3.02105 & 3.02131 & 3.02299 & 3.02337 & 3.02381 & 3.02427 & 3.02554 & 3.02554 \\
\hline 1 & 3.70519 & 3.70508 & 3.71077 & 3.71171 & 3.71277 & 3.71388 & 3.718281 & 3.7183 \\
\hline
\end{tabular}

Table 2. Comparison of $U_{2,4}$ given by OHAM, HAM (q-HAM ; $\left.n=1\right)$, q-HAM $(n=5,20,50,100)$ and $U_{2,6}$ of by q-HAM $(n=100)$ with the solution $u_{2}$

\begin{tabular}{|c|c|c|c|c|c|c|c|c|}
\hline$t$ & $\begin{array}{l}U_{2,4} \\
\text { OHAM }\end{array}$ & $\begin{array}{l}U_{2,4} \\
\quad(n=1 ; h \\
\quad=-0.9326\end{array}$ & $\begin{array}{l}U_{2,4} \\
(n=5 ; h \\
=-4.7\end{array}$ & $\begin{array}{l}U_{2,4} \\
\quad(n=20 ; h \\
=-19.01\end{array}$ & $\begin{array}{l}\boldsymbol{U}_{2,4} \\
\quad(\boldsymbol{n}=50 ; \boldsymbol{h} \\
\quad=-48.001\end{array}$ & $\begin{array}{l}U_{2,4} \\
(n=100 ; h \\
=-96.25\end{array}$ & $u_{2}$ & $\begin{array}{l}U_{2,6} \\
(n=100 ; h \\
=-96.25\end{array}$ \\
\hline 0 & -1 & -1 & -1 & -1 & -1 & -1 & -1 & -1 \\
\hline 0.2 & -1.02136 & -1.02139 & -1.02139 & -1.02139 & -1.02139 & -1.02139 & -1.0214 & -1.0214 \\
\hline 0.4 & -1.09146 & -1.09151 & -1.09155 & -1.09161 & -1.09167 & -1.09169 & -1.09182 & -1.09183 \\
\hline 0.6 & -1.22076 & -1.22084 & -1.22105 & -1.22136 & -1.22164 & -1.22172 & -1.22212 & -1.22213 \\
\hline 0.8 & -1.42229 & -1.4224 & -1.42303 & -1.42395 & -1.42478 & -1.425 & -1.42554 & -1.4256 \\
\hline 1 & -1.71228 & -1.71253 & -1.71398 & -1.71608 & -1.71799 & -1.71849 & -1.718281 & -1.71847 \\
\hline
\end{tabular}


Example 3.2. Consider the following problem of nonlinear integro-differential equation [29]

$$
u^{\prime}(t)=-1+\int_{0}^{t} u^{2}(s) d s, u(0)=0, t \in[0,1]
$$

Assume that $L[\varnothing(t, q)]=\frac{\partial \emptyset(t, q)}{\partial t}$. From (13) the nonlinear operator is

Let $u_{0}(t)=-t$.

$$
[\varnothing(t, q)]=\frac{\partial \emptyset(t, q)}{\partial t}+1-\int_{0}^{t} \emptyset^{2}(s, q) d s .
$$

According to Eq. (2) and Eq. (7) with $R_{m-1}(t)=u_{m-1}^{\prime}+\left(1-\frac{k_{m}}{n}\right)-\int_{0}^{t} \sum_{i=0}^{m-1} u_{i}(s) u_{m-1-i}(s) d s$ the solution Eq. (7) for $m \geq 1$ becomes

Hence, we obtain

$$
u_{m}(t)=k_{m} u_{m-1}(t)+h \int R_{m-1}(t) d t+b,
$$

$$
\begin{aligned}
& u_{1}=-\frac{h t^{4}}{12} \\
& u_{2}=-\frac{1}{12} h n t^{4}+h\left(-\frac{h t^{4}}{12}-\frac{h t^{7}}{252}\right),
\end{aligned}
$$

Then the series solution of q- HAM is

$$
u(t, n, h) \cong U_{M}(t, n, h)=\sum_{k=0}^{M} u_{k}(t, n, h)\left(\frac{1}{n}\right)^{k}
$$

It is found that

$$
\begin{aligned}
\triangle_{1} & =\frac{1}{63}+\frac{h^{4}}{31912704 n^{4}}+\frac{205 h^{3}}{4852224 n^{3}}+\frac{31531 h^{2}}{1769040 n^{2}}+\frac{127 h}{3780 n} \\
\triangle_{2} & =\frac{1}{63}+\frac{297235061 h^{8}}{8398362595392000 n^{8}}+\frac{173605561 h^{7}}{632134819008000 n^{7}}+\frac{9482512903 h^{6}}{195660777312000 n^{6}}+\frac{102775727 h^{5}}{372687194880 n^{5}} \\
\quad+\frac{8547857 h^{4}}{418279680 n^{4}}+ & \frac{1070983 h^{3}}{14152320 n^{3}}+\frac{173 h^{2}}{1620 n^{2}}+\frac{127 h}{1890 n}
\end{aligned}
$$

$\Delta_{m}(t),(m=3,4, \ldots)$ can be calculated similarly.

Figures 10 -14 show the $h$-curves of the square residual error $\Delta_{m}$ given by $\left(1^{\text {th }}, 2^{\text {th }}, 3^{\text {th }}, 4^{\text {th }}\right)$ order approximation to determine the valid region of $h$. The values of $h$ that corresponds to the minimum of the square residual errors is an optimal values of $h$. Table 3 shows the comparison of OHAM), HAM and q-HAM at different values of $n>1$. Clearly, the q-HAM $n>1$ has more accuracy than HAM and OHAM.

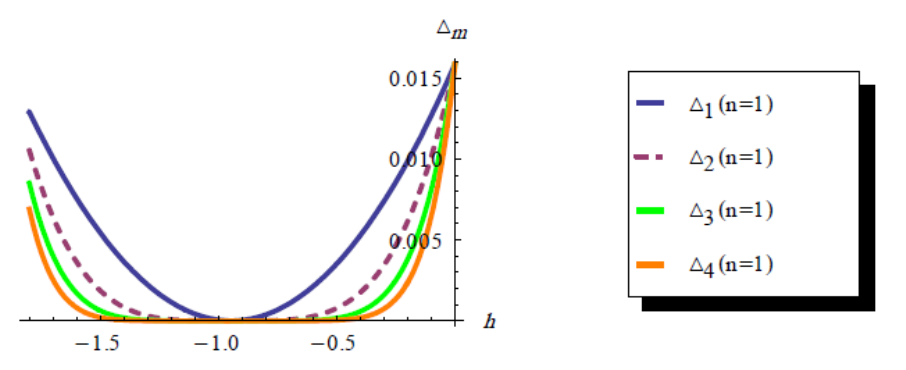

Figure 10. The residual errors $\Delta_{m}(m=1,2,3,4)$ of HAM.

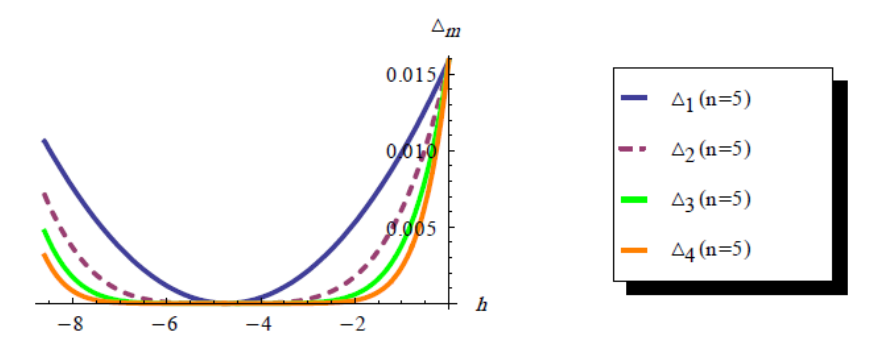

Figure 11. The residual errors $\Delta_{m}(m=1,2,3,4)$ of q-HAM $(n=5)$. 

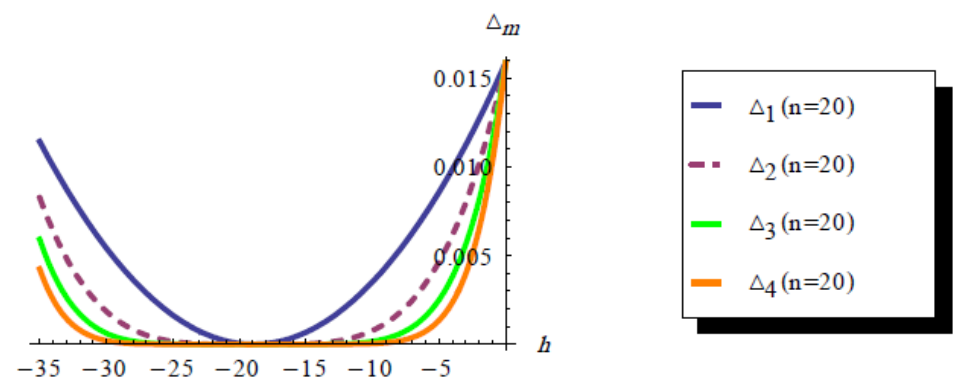

Figure 12. The residual errors $\Delta_{m}(m=1,2,3,4)$ of q-HAM $(n=20)$.
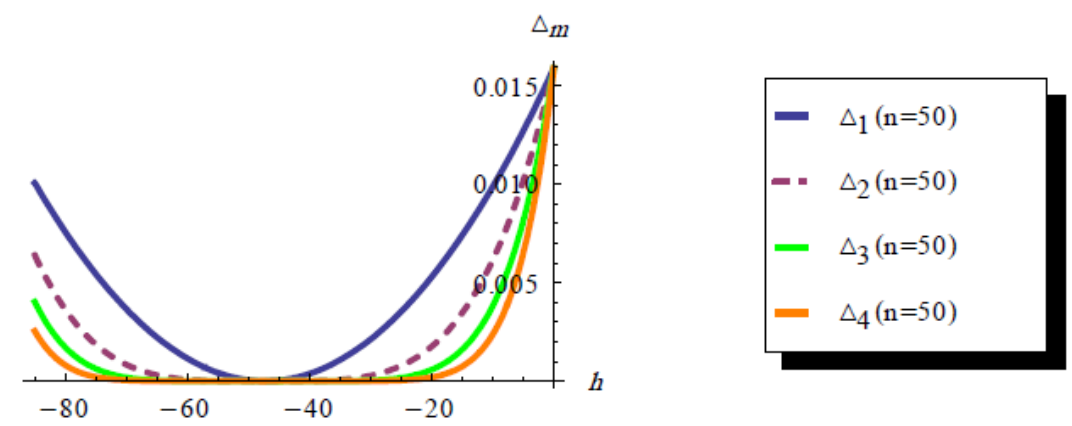

Figure 13. The residual errors $\Delta_{m}(m=1,2,3,4)$ of q-HAM $(n=50)$.

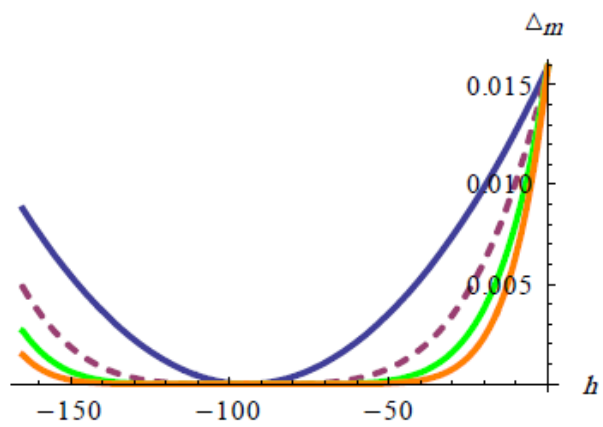

$$
\begin{array}{ll}
\text { - } & \Delta_{1}(\mathrm{n}=100) \\
- & \Delta_{2}(\mathrm{n}=100) \\
- & \Delta_{3}(\mathrm{n}=100) \\
- & \Delta_{4}(\mathrm{n}=100)
\end{array}
$$

\begin{tabular}{|c|c|c|c|c|c|c|}
\hline$m$ & $E_{m}(O H A M)$ & $\Delta_{m}\left(n=1 ; h_{m}\right)$ & $\Delta_{m}\left(n=5 ; h_{m}\right)$ & $\Delta_{m}\left(n=20 ; h_{m}\right)$ & $\Delta_{m}\left(n=50 ; h_{m}\right)$ & $\Delta_{m}\left(n=100 ; h_{m}\right)$ \\
\hline 1 & $0.588916 e-5$ & $\begin{array}{c}0.470089 e-5 \\
-0.942067\end{array}$ & $\begin{array}{c}0.466546 e-5 \\
-4.745\end{array}$ & $\begin{array}{c}0.455403 e-5 \\
-18.87\end{array}$ & $\begin{array}{c}0.453548 e-5 \\
-47.38\end{array}$ & $\begin{array}{c}\text { 0.447006e-5; } \\
-94.567\end{array}$ \\
\hline 2 & $0.182630 \mathrm{e}-8$ & $\begin{array}{c}0.161694 \mathrm{e}-8 \\
-0.965\end{array}$ & $\begin{array}{c}0.1553741 \mathrm{e}-8 \\
-4.849\end{array}$ & $\begin{array}{c}\text { 0.14836e-8; } \\
-19.39\end{array}$ & $\begin{array}{c}0.132012 \mathrm{e}-8 \\
\quad-48.42\end{array}$ & $\begin{array}{c}\text { 0.128842e-8; } \\
-96.75\end{array}$ \\
\hline 3 & $0.247549 \mathrm{e}-12$ & $\begin{array}{c}\text { 0.229395e-12; } \\
-0.9717\end{array}$ & $\begin{array}{c}0.211484 \mathrm{e}-12 \\
-4.86\end{array}$ & $\begin{array}{c}0.199042 \mathrm{e}-12 \\
-19.445\end{array}$ & $\begin{array}{c}0.185862 \mathrm{e}-12 \\
-48.69\end{array}$ & $\begin{array}{c}0.17559 \mathrm{e}-12 \\
-97.32\end{array}$ \\
\hline 4 & $0.671736 \mathrm{e}-16$ & $\begin{array}{c}\text { 0.832667e-16; } \\
\quad-0.9746\end{array}$ & $\begin{array}{c}0.659195 \mathrm{e}-16 \\
-4.8862\end{array}$ & $\begin{array}{c}0.555112 \mathrm{e}-16 \\
-19.5257\end{array}$ & $\begin{array}{c}0.277556 \mathrm{e}-16 \\
-48.882\end{array}$ & $\begin{array}{c}0.346945 \mathrm{e}-17 \\
-97.6296\end{array}$ \\
\hline
\end{tabular}

Figure 14. The residual errors $\Delta_{m}(m=1,2,3,4)$ of q-HAM $(n=100)$.

Table 3. Comparison between residuals of OHAM, HAM and q-HAM $(\boldsymbol{n}>1)$

\section{Conclusion}

In this work, we introduced the comparative study of q-HAM with the Liao's optimal HAM (OHAM). We solved two examples to illustrate the differences between these methods. The outcomes show that the q-HAM was better than the OHAM. 


\section{References}

[1] Liao, S. J. (1992). The proposed homotopy analysis technique for the solution of nonlinear problems. Ph.D. thesis, Shanghai Jiao Tong University.

[2] Liao, S. J. (2003). Beyond Perturbation: Introduction to the Homotopy Analysis Method, Chapman \&Hall/CRC, Boca Raton, Fla, USA.

[3] Liao, S. J. (2004). On the homotopy analysis method for nonlinear problems. Applied Mathematics and Computation, Vol. 147, No. 2, pp. 499-513.

[4] Liao, S. J. (2005). Comparison between the homotopy analysis method and homotopy perturbation method. Applied Mathematics and Computation, Vol. 169, No. 2, pp. 1186-1194.

[5] Liao, S. J. (1997). Homotopy analysis method: a new analytical technique for nonlinear problems. Communications in Nonlinear Science and Numerical Simulation, Vol. 2, No. 2, pp. 95-100.

[6] Abbasbandy, S. (2008). Soliton solutions for the 5th-order KdV equation with the homotopy analysis Abbasbandy S., Soliton solutions for the 5th-order KdV equation with the homotopy analysis method. Nonlinear Dynamics, Vol. 51, No. 1-2, pp. 83-87.

[7] Arafa, A. A. M., Rida, S. Z., and Mohamed, H. (2014). An Application of the Homotopy Analysis Method to the Transient Behavior of a Biochemical Reaction Model. Inf. Sci. Lett.3, No. 1, pp. 29-33.

[8] Jafari, H., Saeidy, M., and Firoozjaee, M. A. (2010). The homotopy analysis method for solving higher dimensional initial boundary value problems of variable coefficients. Numerical Methods for Partial Differential Equations, Vol. 26, No. 5, pp. 1021-1032.

[9] Onyejek, O. N. (2014). Solutions of some parabolic inverse problems by homotopy analysis method. International Journal of Applied Mathematical Research, 3(1).

[10] Zhu, S. P. (2006). An exact and explicit solution for the valuation of American put options. Quantitative Finance, Vol. 6, No. 3, pp. 229-242.

[11] Yabushita, K., Yamashita, M., and Tsuboi, K. (2007). An analytic solution of projectile motion with the quadratic resistance law using the homotopy analysis method. J. Phys. A-Math. Theor., 40, 8403-8416.

[12] Akyildiz, F. T. and Vajravelu, K. (2008). Magneto hydrodynamic flow of a viscoelastic fluid. Phys. Lett. A., 372, 3380-3384.

[13] Niu, Z. and Wang, C. (2010). A one-step optimal homotopy analysis method for nonlinear differential equations. Communications in Nonlinear Science and Numerical Simulation, 15, 2026-2036.

[14] Liao, S. J. (2010). An optimal homotopy-analysis approach for strongly nonlinear differential equations. Commun. Nonlinear Sci. Numer. Simulat., 15, 2003-2016.

[15] El-Tawil, M. A. and Huseen, S. N. (2012). The q-Homotopy Analysis Method (q-HAM). International Journal of Applied Mathematics and Mechanics, 8(15): 51-75.

[16] El-Tawil, M. A. and Huseen, S. N. (2013). On Convergence of The q-Homotopy Analysis Method. Int. J. Contemp. Math. Sciences, Vol. 8, No. 10, 481-497.

[17] Huseen, S. N. and Grace, S. R. (2013). Approximate Solutions of Nonlinear Partial Differential Equations by Modified q-Homotopy Analysis Method (mq-HAM). Hindawi Publishing Corporation, Journal of Applied Mathematics, Article ID 569674, 9 pages.

[18] Huseen, S. N., Grace, S. R., and El-Tawil, M. A. (2013). The Optimal q-Homotopy Analysis Method (Oq-HAM). International Journal of Computers \& Technology, Vol 11, No. 8.

[19] Huseen, S. N. (2015). Application of optimal q-homotopy analysis method to second order initial and boundary value problems. Int J Sci Innovative Math Res (IJSIMR), 3(1):18-24.

[20] Huseen, S. N. (2015). Solving the K (2, 2) Equation by Means of the q-Homotopy Analysis Method (q-HAM). International Journal of Innovative Science, Engineering \& Technology, Vol. 2, Issue 8.

[21] Huseen, S. N. (2016). Series Solutions of Fractional Initial-Value Problems by q-Homotopy Analysis Method. International Journal of Innovative Science, Engineering \& Technology, Vol. 3, Issue 1.

[22] Huseen, S. N. (2017). A Numerical Study of One-Dimensional Hyperbolic Telegraph Equation. Journal of Mathematics and System Science, 7, 62-72. 
[23] Huseen, S. N. and Nada M. Ayay. (2018). A New Technique of The q-Homotopy Analysis Method for Solving Non-Linear Initial Value Problems. Journal of Progressive Research in Mathematics, Volume 14, Issue 1, 2292-2307.

[24] Huseen, S. N. and Rawan A. shlaka. (2019). The Regularization q-Homotopy Analysis Method for (1 and 2)-Dimensional Non-linear First Kind Fredholm Integral Equations. Journal of Progressive Research in Mathematics, Volume 15, Issue 3, 2721-2743.

[25] Akinyemi, L. (2019). q-Homotopy analysis method for solving the seventh-order time-fractional Lax's Korteweg-deVries and Sawada-Kotera equations. Comp. Appl. Math., 38(4), 191.

[26] L. Akinyemi, S. N. Huseen (2020). A powerful approach to study the new modified coupled Korteweg-de Vries system. Mathematics and Computers in Simulation, 177, 556-567.

[27] Huseen, S. N. (2020). On Analytical Solution of Time-Fractional Type Model of the Fisher's Equation. Iraqi Journal of Science, Vol. 61, No. 6, pp. 1419-1425.

[28] Huseen, S. N., El-Tawil, M. A., Grace S. R., and Ismail, G. A. (2020). Solving High-Order Nonlinear Partial Differential Equations by Modified q-Homotopy Analysis Method. AJMS/Apr-Jun, Vol. 4, Issue 2, pp. 25-46.

[29] Jafar S. Nadjafi and Hossein S. Jafari. (2011). Comparison of Liao's optimal HAM and Niu's one-step optimal HAM for solving integro-differential equations. Journal of Applied Mathematics and Bioinformatics, Vol. 1, No. 2, 85-98. 